\title{
Summary Report of the First International Workshop on PET/MR Imaging, March 19-23, 2012, Tübingen, Germany
}

Dale L. Bailey, ${ }^{1}$ Henryk Barthel, ${ }^{2}$ Thomas Beyer, ${ }^{3}$ Ronald Boellaard, ${ }^{4}$ Brigitte Gückel, ${ }^{5}$ Dirk Hellwig, ${ }^{6}$ Hans Herzog, ${ }^{7}$ Bernd J. Pichler, ${ }^{8}$ Harald H. Quick, ${ }^{9}$ Osama Sabri, ${ }^{2}$ Klaus Scheffler, ${ }^{10,11}$ Heinz P. Schlemmer, ${ }^{12}$ Nina F. Schwenzer, ${ }^{5}$ Hans F. Wehrl ${ }^{8}$

${ }^{1}$ Department of Nuclear Medicine, Royal North Shore Hospital, Sydney, Australia

${ }^{2}$ Department of Nuclear Medicine, University of Leipzig, Leipzig, Germany

${ }^{3}$ Center for Medical Physics and Biomedical Engineering, Medical University of Vienna, Vienna, Austria

${ }^{4}$ Department of Nuclear Medicine and PET Research, VU University Medical Centre Amsterdam, Amsterdam, The Netherlands

${ }^{5}$ Department of Diagnostic and Interventional Radiology, Eberhard Karls University Tübingen, Tübingen, Germany

${ }^{6}$ Department of Nuclear Medicine, Saarland University Medical Center, Homburg, Saar, Germany

${ }^{7}$ Institute of Neuroscience and Medicine-4, Forschungszentrum Jülich, Jülich, Germany

${ }^{8}$ Division Werner Siemens Imaging Center, Department of Preclinical Imaging and Radiopharmacy, Eberhard Karls University Tübingen, Tübingen, Germany

${ }^{9}$ Institute of Medical Physics, Friedrich-Alexander-University Erlangen-Nürnberg, Erlangen, Germany

${ }^{10}$ Department of Biomedical Magnetic Resonance, Eberhard Karls University Tübingen, Tübingen, Germany

${ }^{11}$ DMRC Department, Max Planck Institute for Biological Cybernetics, Tübingen, Germany

${ }^{12}$ Department of Radiology, German Cancer Research Center (DKFZ), Heidelberg, Germany

\begin{abstract}
We report from the First International Workshop on positron emission tomography/magnetic resonance imaging (PET/MRI) that was organized by the University of Tübingen in March 2012. Approximately 100 imaging experts in MRI, PET and PET/computed tomography (CT), among them early adopters of pre-clinical and clinical PET/MRI technology, gathered from March 19 to 24, 2012 in Tübingen, Germany. The objective of the workshop was to provide a forum for sharing first-hand methodological and clinical know-how and to assess the potential of combined PET/MRI in various applications from pre-clinical research to scientific as well as clinical applications in humans. The workshop was comprised of pro-active sessions including tutorials, specific discussion panels and grand rounds. Pre-selected experts moderated the sessions, and feedback from the subsequent discussions is presented here to a greater readership. Naturally, the summaries provided herein are subjective descriptions of the hopes and challenges of PET/MR imaging as seen by the workshop attendees at a very early point in time of adopting PET/MRI technology and, as such, represent only a snapshot of current approaches.
\end{abstract}

Key words: Combined imaging, Molecular imaging, PET/CT, PET/MRI, Quantification, Artifacts, MR-based attenuation correction, Workflow, Standardization

The authors are listed in alphabetical order and contributed equally to this paper.

Correspondence to: Thomas Beyer; e-mail: thomas.beyer@meduniwien.ac.at 


\section{Introduction}

$\mathrm{M}$ edical imaging using combined multi-modality imaging technologies has continuously improved clinical patient care over the past decade. Recently, a new imaging modality, combined positron emission tomography/magnetic resonance imaging (PET/MRI), has been proposed for application in humans, thus opening the door to true multiparametric imaging [1]. After early attempts to solve the challenges of integrating PET and MRI systems, three general strategies are now conceived for combining PET and MRI [2]: separate PET (i.e., PET/computed tomography (PET/CT) and MRI units, sequential, or co-planar PET/MRI, and fully integrated PET/MRI systems.

As of today, there are about 40 clinical and ten preclinical combined PET/MRI systems installed worldwide. Therefore, exploring any synergistic effects of combining functional MRI and molecular PET imaging would be of great interest. In discussions during the meeting, the view emerged that PET/MRI must offer new areas of application exploiting the strengths of each technology and, as such, is not seen as being in conflict with PET/CT. However, while first clinical studies are currently underway to assess the potential of combined PET/MRI, several methodological challenges still need to be addressed before this imaging technology can be transferred into routine applications.

This workshop was designed specifically to invite professionals from complementary disciplines, such as nuclear medicine and radiology, physics and biology, as well as clinicians and pharmacists. The overarching goal was to reflect the multidisciplinary nature of the combination of imaging hardware by the multi-disciplinary panel of faculty, experts and attendees.

This article combines summaries of the six breakout sessions (BS) from the workshop and subsequent panel discussions, which were either moderated or supported by the authors of this report. The topics of the breakout sessions were chosen ahead of the workshop by the organizers of the meeting in discussions with some workshop attendees and most of the speakers. In this summary report, we review the specific roles of both MRI and PET in combined PET/MRI. Further, we address the need for quantitative imaging, including mandatory corrections (e.g., attenuation, scatter, normalization, motion). This leads to a more detailed perspective on the need for, and application of, quantitative PET and MRI in the context of PET/MRI and which level of quantitative accuracy is required for clinical and research applications. Finally, in light of the use of PET/MRI for clinical research, the requirements for standardization of PET/MRI are discussed.

This workshop did not intend to promote one PET/MRI design over another since sufficient clinical and methodological data to validate such a potential preference are missing today. Furthermore, the issue of cost was not addressed as this technology is still in an early exploratory phase and future designs may well be quite different to current versions.

\section{BS 1: the Role of MRI in PET/MRI}

The role of MRI in PET/MRI strongly depends on the preselected application of PET/MRI. For example, PET/MRI can be used in research applications, where access to the unbiased spectrum of MR-only techniques is desirable, much like the ability to perform dynamic emission acquisitions, while time constraints as well as diagnostic demands are less important. Alternatively, PET/MRI can be used clinically, with the main objective of the examination being diagnostic, thus, giving priority to other parameters and rendering a short examination time desirable.

In either scenario, the choice of MR sequences and the body region(s) covered by MRI determine the total time of an examination. MR images are used for anatomical localization, attenuation correction of PET data and tissue characterization based on selected functional MRI (fMRI) techniques.

As of today, two approaches towards combined PET/MRI are available [2]: first, a sequential approach, with the PET and MR data being acquired in close sequence in time (similar to $\mathrm{PET} / \mathrm{CT}$ ), and second approach. Here, the PET detector is fully integrated into the MR system, and PET and MR data can be acquired simultaneously.

Approaches to both hardware combinations deal with the challenge of choosing the appropriate MR sequences on top of the mandatory sequences for deriving the attenuation coefficients [3]. A PET/MRI protocol can be performed either without further 'morphological' MRI, thus taking about $25 \mathrm{~min}$, or with a number of additional, diagnostic sequences, even with the use of intravenous (i.v.) contrast, thus increasing the MRI acquisition time to 90 min or more. In this context, it is mandatory to tailor individual MRI protocols to the specific patient and diagnostic question to be answered, thereby streamlining the data acquisition workflow and shortening the acquisition time. For true total body and torso imaging, previous standard multi-station, multi-contrast body part MRI should be replaced by multi-contrast continuously moving table data acquisition schemes [4].

Integrated PET/MRI systems also allow for physiological gating (e.g., by using electrocardiography) as well as MRbased triggering of the PET data. It was discussed that motion correction techniques need further improvements to increase the acceptance level and clinical applicability of MR-based PET motion correction. Therefore, one requirement is to limit the associated increase in examination time when applying gating methods.

Further considerations relate to the large amount of data that is generated and that needs to be stored and handled, which, in turn, requires sophisticated software solutions for efficient reading dynamic and quantitative assessments.

Finally, reimbursement issues and imaging costs were discussed as well. Without a doubt, both have a major impact on the choice of the clinically viable workflows and, therefore, will in part determine whether PET/MRI is going to become a high-throughput imaging modality or brand itself into a research modality. 
BS 1. The role of MRI in PET/MRI

Key Questions

- How could we structure the examination protocols for PET/MRI for optimal diagnostic information in a minimum amount of time?

- Are there MR sequences which can be improved or adapted to the needs of PET/MRI and workflow?

- What are the best software solutions and approaches for convenient and reliable data storage?

Status Quo

- Two general approaches towards combined PET/MRI are available (simultaneous and sequential) facing similar problems, i.e., choice and amount of MRI sequences.

- There are several possibilities to create PET/MRI protocols depending on the choice of MR sequences, leading to examination times between 25 and $90 \mathrm{~min}$ or longer.

- MRI currently is performed in a multi-station, multi-contrast weighting fashion requiring time-consuming examination planning for wholebody imaging, thus resulting in non-optimized workflow.

- MR-based motion correction is promising in fully integrated systems with several limitations. Further developments will have an influence on the acceptance of MR-based motion corrections in clinical routine examinations.

Conclusions and Open Questions

$>$ The amount of MRI performed depends on the underlying pathology, the clinical work-up as well as patient tolerance.

$>$ MRI protocol and imaging workflow optimization is required since MRI is the limiting factor. This may involve the development and integration of easy-to-use moving table data acquisition strategies.

$>$ Reimbursement will also influence the PET/MRI workflow since it has a major impact on the patient throughput.

\section{BS 2: the Role of PET in PET/MRI}

One prerequisite for the combined PET/MRI technique gaining wider acceptance in the medical imaging community is the PET sub-system delivering data of similar quality as that obtained with PET data using PET/CT or stand-alone PET systems. This holds true for physical properties like spatial resolution, scatter fraction and, most importantly, accurate radioactivity quantification and standardized uptake value (SUV). For example, the event counting rate and sensitivity performance of an avalanche photodiode (APD)-based MRI-integrated PET detector system with an extended $26-\mathrm{cm}$ axial field-ofview and $60-\mathrm{cm}$ ring diameter, both of which contribute to improved sensitivity, can be equal to that of a stateof-the-art photomultiplier tube-based system or even exceed its performance [5].

With regard to clinical applications, different artifacts in attenuation correction (AC) maps are produced by combined PET/MRI compared to PET/CT, most of them resulting from inaccuracies in the MRI-based AC (e.g., metal implants, misclassification of tissue, truncation). Those occasional artifacts were reported to almost exclusively occur in the head, torso and extremities and have to be checked for. Importantly, bony structures are not considered by the currently applied MR-based AC method (three- and four-class segmentation), resulting in a systematic undercorrection of the PET data $[3,6]$. This is particularly important for neuroscience studies considering that the brain is surrounded by the bony structures of the skull.
However, the determination of the resulting error is still the subject of ongoing studies.

Overall, the PET image quality and alignment with MR is subjectively rated high by clinical observers, and the diagnostic value is comparable with that of standard PET/CT systems. The known differences in SUVs obtained by combined PET/MRI as compared to PET/CT require consideration and further research as they might affect the usefulness of combined PET/MRI in the evaluation of disease progression and therapy response.

In routine clinical studies, combined PET/MRI has the opportunity to improve patient comfort and enhance throughput compared with sequential PET and MRI examinations. Figure 1 provides a "MindMap" for the clinical role of PET/MRI developed in real time by the participants of this breakout session. It is evident that the spectrum of expected clinical applications is wide and covers different fields in oncology, cardiology and neuroscience imaging.

The experience from PET/CT has been that the speed of the CT scan, the derivation of CT-based attenuation and scatter correction algorithms for PET data and the anatomical localization afforded by the CT images are all beneficial and provide essential information from the CT to supplement the PET data. CT, however, has largely failed to be enhanced from the inclusion of co-acquired PET data, except in the area of clinical image interpretation. Due to the different basis for generating signals in MRI though, it may be that PET and MRI both contribute useful information to the other modality such as glucose metabolic rate (FDG-PET) and MR spectroscopy of metabolic substrates or products in a way that has not occurred with PET/CT.

Further, for a number of scientific questions, the use of simultaneous dynamic PET/MRI is potentially preferable over independent or sequential PET and MRI acquisitions when:

(a) Conventional, non-invasive diagnostic imaging of acute and changing perfusion conditions of the heart and the brain (e.g., stress cardiac imaging, acetazolamide-induced changes in rCBF) by use of contrast-enhanced MRI should be cross-validated by truly simultaneous perfusion PET.

(b) Kinetic PET data modeling should be performed without invasive arterial blood sampling by using a PET/MRIderived arterial input function.

(c) Functional brain MRI studies, which rely on the blood oxygen level dependent (BOLD) effect and which, for instance, display brain response to certain tasks or pharmacologic agents are paralleled by PET measurements of neurotransmission to improve the understanding of a number of different brain disorders.

(d) The nature of the BOLD contrast itself and the neuro-vascular coupling is further elucidated by simultaneous measurement of glucose metabolism or cerebral perfusion in PET.

Finally, the exact knowledge of body composition and organ movement through simultaneous MRI holds the potential to improve movement correction and the accuracy of measured SUVs in oncologic PET. 
Many workshop attendees expressed their hopes that simultaneous PET/MRI, more so than sequential PET/MRI, would be preferred in selected clinical scenarios when the total examination time is of essence. In young children, for example, fully integrated PET/MRI could help reduce the total examination time and, therefore, the need for, or duration of, sedation and anesthesia. Also, in an uncooperative patient, simultaneous examination has distinct advantages.

BS 2. The role of PET in PET/MRI

Key Questions

- Is the quality of the PET component sufficient for qualitative and quantitative imaging?

- Is the simultaneous acquisition of (dynamic) PET and MRI necessary in clinical routine?

Status Quo

- The physical parameters of the PET sub-systems of combined PET/MRI are reported to be satisfactory. Artifacts different from PET/CT occur occasionally (mostly related to MR-based AC).

- SUVs of PET-positive lesions can be reduced compared to PET/CT due to the inaccurate representation of bone attenuation values in MR-AC.

- For a number of important research questions in neuropsychiatry, cardiology and oncology, simultaneous PET/MRI is potentially preferable.

Conclusions and Open Questions

$>$ Despite lower SUVs, the diagnostic quality of PET in PET/MRI is not impaired compared to PET/CT.

$>$ The systematic error of PET data following MR-AC needs to be assessed further for currently employed three- or four-class segmentation methods.

$\triangleright$ As far as can be concluded so far, especially imaging of brain disorders, soft tissue cancers, cancers in the proximity of moving organs, and pediatric cancers will profit from combined and simultaneous PET/MRI.

$>$ Fully integrated PET/MR may further benefit the assessment of cancers in the proximity of moving organs.

$>$ Future research will also focus on potential other clinical and research applications, for instance, in cardiology.

\section{BS 3: Translational Aspects}

The origins of PET/MRI research were in the pre-clinical field, where first developments and applications date back to the late 1990s [7-10]. At about the same time, important progress towards APD-based PET detectors was made by the Munich group [11].

The pre-clinical imaging arena is an important field to support subsequent clinical applications. Animal models can be used to generate specific responder and nonresponder models for diseases and to provide data for efficient drug development. Combined and fully integrated PET/MRI systems are potentially helpful in this context. For example, minute amounts of labeled drugs can be tracked using high-sensitivity PET, while pharmacodynamic changes or physiological effects (e.g., perfusion) induced by the drug can be measured at the same time using arterial spin labelling. It is also feasible to assess the metabolic profile of the brain during drug application by MR spectroscopy while observing changes in brain receptor status using PET at the same time. Control conditions (e.g., age of animals, nutrition, environmental factors, etc.) allow an exact characterization of disease pathways such as in oncology or neurology. In vivo imaging methods can be easily augmented with ex vivo methods, such as histology, mass imaging or metabolic profiling. Here, spectrometry imaging, such as matrixassisted laser desorption ionization and secondary ion mass spectrometry, allows a spatially resolved profiling of biomolecules by applying, e.g., nuclear magnetic resonance spectroscopy in tissue samples, which is a clear advantage when studying diseases in animal models. In addition, animal studies can be used to estimate radiation exposure (e.g., internal dosimetry) from newly developed PET tracers for application in humans.

Simultaneity of PET and MRI signal detection is highly desirable for pre-clinical PET/MRI and advanced research applications. Compared to humans, small animals express accelerated physiological parameters such as heart rate or respiration and even metabolism. Therefore, an isochronous acquisition of multifunctional imaging parameters, such as perfusion using MRI and oxygenation using PET, is even more important since fluctuations and changes of these parameters occur on a smaller timescale, thus making a correlation of sequential measurements challenging.

Another benefit of PET/MRI over PET/CT in small animals is the reduced radiation exposure of the animals. In treatment models, where follow-up studies using multiple time points are desired, repetitive CT measurements can deposit a substantial amount of radiation dose to the animal, which may even alter experimental outcomes [12]. Small animal CT doses depend on image resolution and contrast and can easily add up to 5 Gy per mouse [13], while the absorbed dose in mice undergoing a small animal PET study is on the order of 0.9 Gy [14]. Thus, PET radiation doses are an order of magnitude lower than those from small animal CT scans. Another advantage of small animal MR over CT is also the improved soft tissue characterization, which can be obtained also, albeit with somewhat limited success, by using i.v. contrast agents, which, in turn, may affect the animal model in study. There was a consensus among the workshop attendees that sequential PET/MRI appears to be suitable for the pre-clinical field, however, only if the main focus of the studies is to compare PET-based function with MR-based anatomy.

Finally, dedicated studies are required to validate the accuracy and reproducibility of pre-clinical PET/MRI experiments. In line with clinical PET/MRI, issues like MR-based AC are important also for small animal PET/ MRI. Multi-center comparability should be encouraged by dedicated PET/MRI phantoms as part of a standardized quality assurance procedure. The development of pre-clinical PET/MRI protocols should also center on hallmarks important for clinical imaging, i.e., high throughput and robust methods and protocols. However, dedicated studies of complex functions such as the underpinnings of the BOLD-fMRI effect might require 


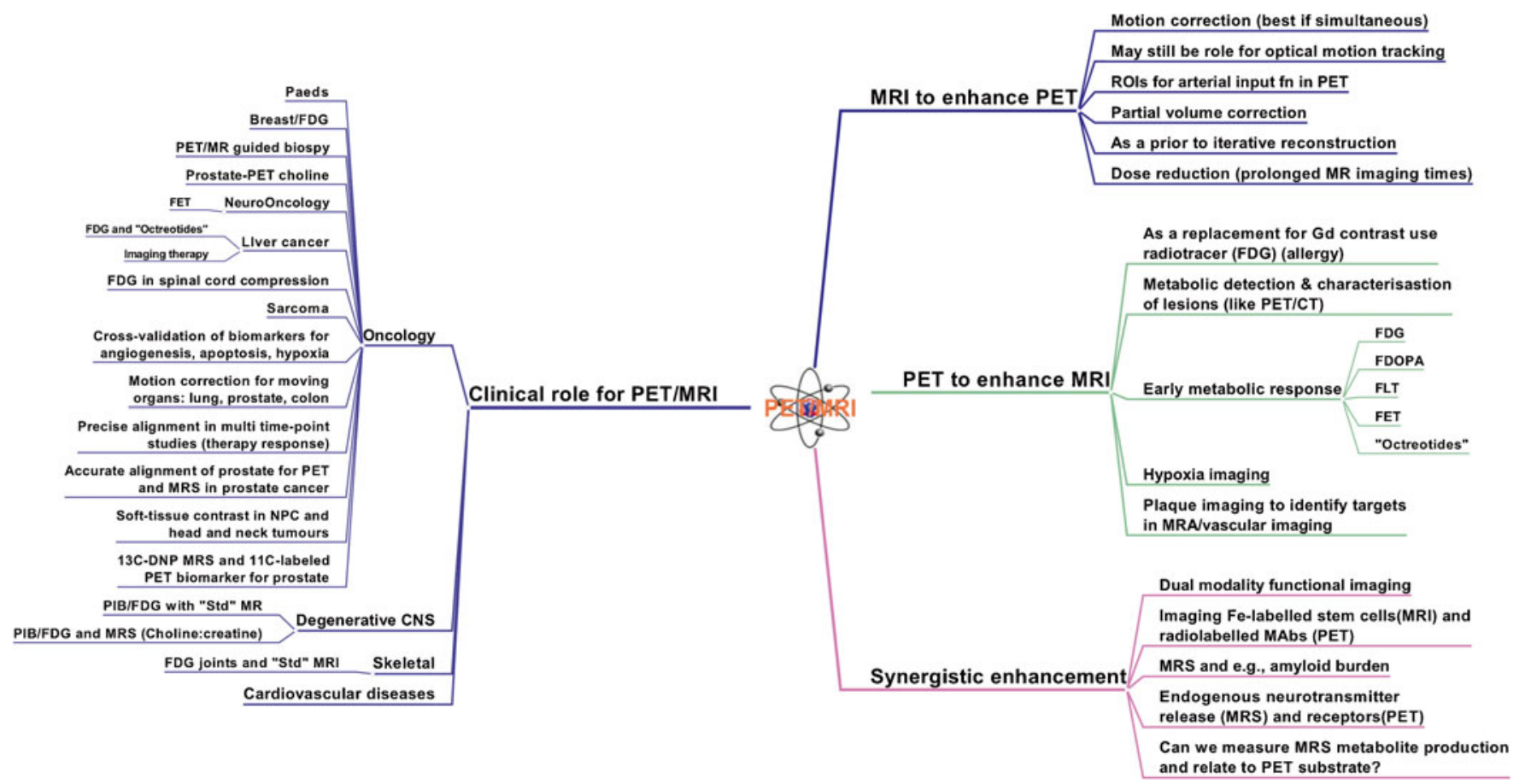

Fig. 1. "MindMap" on the clinical role of PET/MRI developed in real time by the participants of the breakout session BS 2 "The role of PET in PET/MRI".

more elaborate protocols, which are easier to realize in a pre-clinical setting.

BS 3. Translational Aspects

Key Questions

- Is pre-clinical PET/MRI beneficial for small animal research?

-What are the advantages of pre-clinical studies compared to clinical studies?

- Which PET/MRI system design is most useful for pre-clinical imaging today? Status Quo

- PET/MRI offers many advantages such as reduced radiation dose, functional parameters and improved tissue characterization compared to pre-clinical PET/CT.

- First pre-clinical PET/MRI systems are already in use.

- Fast physiology in animals as well as higher throughput favour simultaneous PET/MRI.

Conclusions and Open Questions

$>$ Studies are needed to show the quantitative accuracy and reproducibility of small animal PET/MRI.

$>$ Complex research questions can be studied more easily using the controlled conditions of pre-clinical research.

$>$ Sequential approaches are suitable to combine anatomy with function; however, multifunctional research questions are more likely to be answered using isochronous PET/MRI.

$>$ Translation mandates interdisciplinary training for users.

$>$ Funding and cost-benefit models will impact the progress of preclinical PET/MRI.

\section{BS 4: Correction Methods}

A key objective of combined PET-based hardware fusion systems is to maintain the ability to fully quantify metabolic pathways non-invasively. PET quantification by means of SUV or more advanced kinetic modeling is required for clinical imaging of patients for primary diagnosis and therapy response assessment as well as for new tracer developments. Likewise, quantification of dedicated MR signals such as apparent diffusion coefficient, perfusion, etc., must be supported in the context of combined multifunctional PET/MRI (Fig. 2).

Accurate attenuation correction is a prerequisite for quantitative PET imaging. Furthermore, scatter correction, a second important correction in PET imaging, may depend on accurate transmission information. In PET/MRI, the PET attenuation coefficients are derived from MR images that are not representative of electron density variations, unlike in CT and standard PET transmission scanning. Several approaches deriving attenuation coefficients from MR images have been proposed and are well summarized in [15]. Routine applications of MR-based AC, available in whole-body PET/MRI, are based on the acquisition of dedicated MR sequences (such as the DIXON sequence with in-/opposed phase images) and subsequent segmentation. Alternative algorithms, targeted towards research applications, apply AC templates in the case of brain PET/ MRI or aim at combining tissue segmentation with atlas approaches. However, these techniques may lack reproducibility in situations with non-standard patient anatomy. Recent theoretical work by Defrise et al. suggests that time-of-flight information from the PET measurement may have a role to play in improving the accuracy of MRIderived AC maps [16]. 

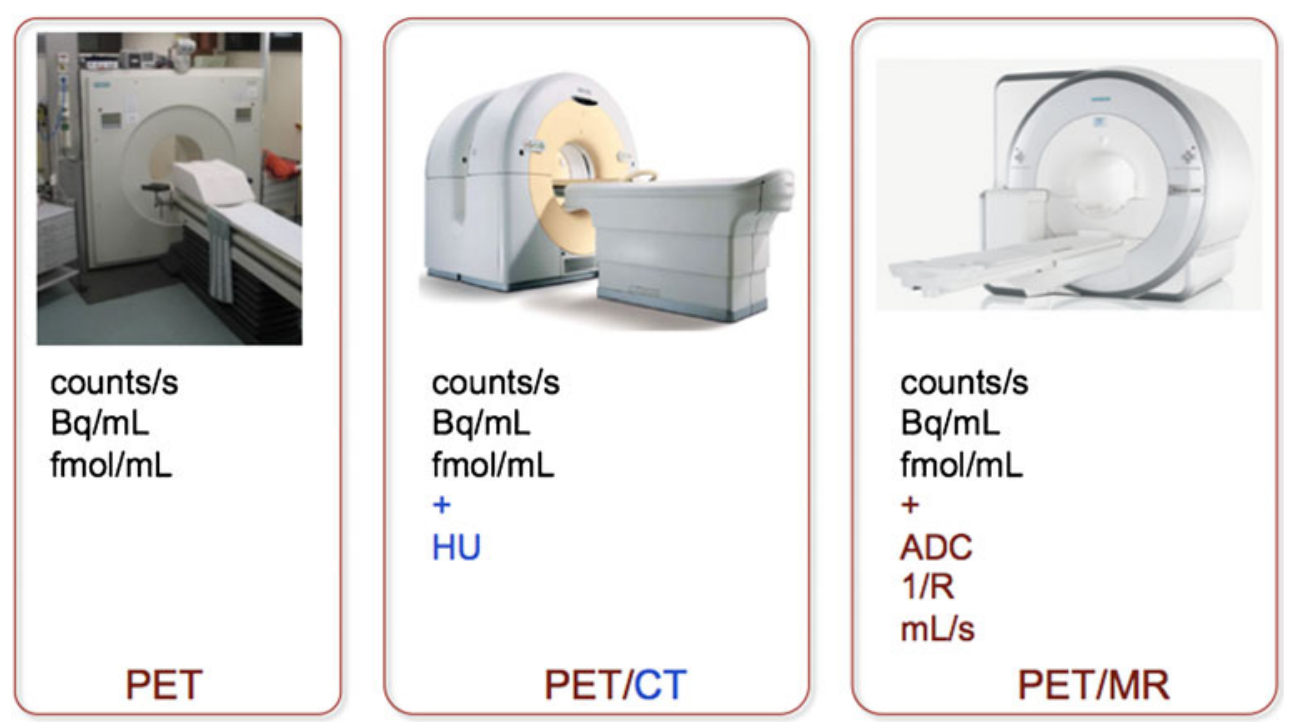

Fig. 2. Quantitative parameters in PET, PET/CT and PET/MR.

In addition to the reproducibility of a selected MR-AC method, the absolute accuracy is a matter of debate. Interestingly, the acceptance level among the attendees of the breakout session varied between $0 \%$ and $20 \%$, depending on the anticipated PET/MRI application and the personal involvement of the interviewed expert in research applications. When discussed further in detail, the acceptance level was considered to be adjustable to the area of interest, meaning that as long as the absolute bias is known a priori, it would be acceptable. This can be seen as somewhat contradictory to the past when some researchers argued strongly in favour of the non-invasive imaging and quantification ability of PET. Today, the gold standard for quantitative accuracy of PET/MRI is PET/CT (i.e., CT-based AC), mainly due to the fact that almost no PET-only systems with standard PET transmission sources are produced anymore. Furthermore, in clinical PET where the diagnosis is often based on a qualitative evaluation, the accuracy of quantification may not be crucial, if residual errors do not distort image quality. In contrast, brain PET/MRI studies combining, e.g., PET-based neuroreceptor imaging with fMRI, or PET/MRI-based therapy response assessment in oncology disease requires accurate PET quantification, which is, however, not sufficiently supported by the software in commercial PET/MRI systems today.

In addition to tissue attenuation, other sources of attenuation must be considered. These were discussed as being surface coils, additional positioning devices, MR contrast agents and the like. Most of these contributions are understood and corrections (look-up tables) are available to account for these effects. On the other hand, while truncation artifacts, arising from the difference in transverse field-of-view of the PET and MRI, are known from PET/CT imaging and understood, no fully satisfactory correction method is yet available in present PET/MRI systems.

Figure 3 summarizes the mandatory corrections in PET/ MRI in the context of the required corrections for either modality alone. It is clear that, with the integration of PET and
MRI, the number of corrections and their complexity increased. Most of these corrections (attenuation, scatter and truncation) are understood today, and first implementations are promising yet not perfect. Quantitative accuracy is in most clinical cases as good as in clinical PET/CT, but the reproducibility is lower. On the upside, the prospects of using the MRI information for super-corrections of the PET (e.g., motion, partial volume effect) are big, but practical solutions will require the successful allocation of a few key applications of PET/MRI in routine imaging. Otherwise, advanced correction schemes will remain a niche for clinical researchers.

BS 4. Correction Methods

Key questions

- Which of the available methods for MR-AC is acceptable for clinical routine?

- What is the level of bias from MR-AC we are prepared to accept in clinical practice?

Status Quo

- Several approaches to MR-AC are available in routine PET/MRI systems, while being an active field of ongoing research.

- There is no accepted correction method for metal implants and bone implemented in PET/MRI.

- First solutions to truncation artifacts are made available, but further work is needed.

Conclusions and Open Questions

$\triangleright$ MR-based motion correction, if implemented correctly, is likely to accelerate the adoption of PET/MRI.

$\triangleright$ MR-based correction for PET is very promising (e.g., partial volume correction, model-guided reconstruction).

$>$ Corrections for MR non-uniformities are required (depending on choice of sequence).

\section{BS 5: Standardization and Clinical Trials}

The main purpose of therapeutic clinical trials is to determine the efficacy of a new treatment. In cancer trials, the 'ideal' measure for treatment efficacy is the final 


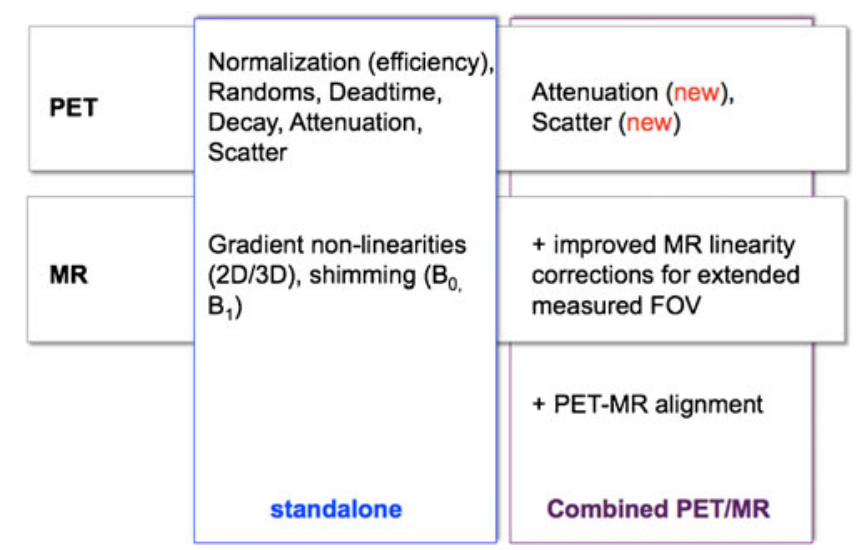

Fig. 3. Mandatory corrections and quality control measures in PET only, PET/CT, and PET/MR imaging.

outcome based on overall survival. Yet, in practice, overall survival is often not to be used because of the possibly extended life expectancy of patients and hence prolonged duration of the trial. In these cases, patients will undergo other treatments after the trial is concluded and, consequently, overall survival is affected not only by the drug under evaluation but also by other treatments. Furthermore, early response monitoring and response prediction is particularly important as quality of life and survival have to be considered. In addition, the high cost of a new treatment ideally requires prediction of response early after the onset of therapy under investigation.

Therapeutic clinical trials frequently rely on surrogate endpoints. A surrogate endpoint, or 'biomarker', is a laboratory or image-based measurement or physical sign used in therapeutic trials as a substitute for a clinically meaningful endpoint (e.g., overall survival) that is a direct measure of how a patient feels, functions or survives and is expected to predict the effect of the therapy. There are several biomarkers at hand that may act as surrogate endpoints. Imaging biomarkers are physical entities or images of these entities that can be measured and used to indicate a biological process, disease process or drug response. CT, MRI, PET and $\mathrm{PET} / \mathrm{CT}$ are nowadays used in various ways in clinical trials.

The use of morphologic CT as biomarker was first established by the publication of the Response Evaluation Criteria in Solid Tumors (RECIST) [17, 18]. The RECIST criteria were established by an international collaboration, including the European Organisation for Research and Treatment of Cancer (EORTC), National Cancer Institute of the United States and the National Cancer Institute of Canada Clinical Trials Group. These criteria represent a set of rules that define when cancer patients improve ("respond"), stay the same ("stabilize") or worsen ("progression") during treatments based on tumour size changes seen on CT. Almost at the same time in 1999, Young et al. published the EORTC 2-deoxy-2-[ ${ }^{18}$ F]fluoro-D-glucose ([18F]-FDG) PET response criteria [19]. In 2009, Wahl et al. published a paper entitled "From RECIST to PERCIST: evolving considerations for PET response criteria in solid tumors", providing recommendations for, amongst others, ${ }^{18}$ F-FDG PET image analysis and response evaluation [20].

Ideally, biomarkers, including those based on imaging, should not only indicate a biological process, disease process or drug response, but they should also provide accurate and reproducible quantitative measures of these processes. Precision is of utmost importance as unwanted variability in (imaging) results may obscure or underestimate the efficacy of the new drug. Therefore, standardization of imaging procedures is essential in clinical trials, particularly multi-center clinical trials [21, 22]. The level and type of standardization may, however, depend on the specific utility of the imaging test during the trial. Imaging can be used to determine patient eligibility to participate in the trial (based on staging or disease severity), stratify patients into various trial arms or measure treatment response during or after the treatment.

During the breakout session on this topic, there was general consensus that when imaging is used for treatment response assessment intra-subject standardization of imaging procedures and harmonization of imaging equipment performance is essential. This is of importance not only for PET but also for other functional imaging methods, such as CT or MRI perfusion, MRI diffusion and MRI spectroscopy. When using imaging to obtain absolute measures, however, higher standards are required, such as inter-subject or even cross-center standardization. However, these standards were considered difficult to achieve at best.

Several issues that may hamper these harmonization efforts were raised, such as:

- Ongoing technological advances are more quickly introduced than standards.

- Several organizations have similar yet different standards and system calibration/validation programs.

- Imaging system vendors have not yet implemented acquisition, reconstruction and analysis protocols or tools resulting in harmonized imaging results.

A pragmatic solution for trials could be to define standards for the execution of the imaging procedure in combination with a minimal performance standard for the equipment (verified by quality control experiments) and with centralized reading and analysis of the CT, MRI, PET or PET/CT images. Central analysis by an imaging core laboratory could include post-processing steps to harmonize quantitative results derived from these images. Finally, it was acknowledged that despite the present 
(large) variability in imaging procedures [23], CT, MRI, $\mathrm{PET}$ and PET/CT have made major contributions in improving patient diagnostic accuracy together with an impact on patient management [24]. Despite the fact that the PET/MRI technology is still evolving, it has a great potential to provide unique information and quantitative parameters for therapy response monitoring in clinical trials.

BS 5. Standardization and Clinical Trials

Key questions

- Is there a need for (imaging) standardization in clinical trial (and in clinical practice)?

- How to agree on standards in multi-center trials? Who sets the standards?

- Do we need different standards for single versus multi-center trials?

Status Quo

- There is a large variability in applied methodology of imaging examinations.

- Standards and QC experiments are set up by scientific societies, clinical research organizations and collaborative groups, resulting in different standards.

- Present PET/CT and PET/MRI system vendors do not provide standardized acquisition, reconstruction and analysis protocols resulting in harmonized image quality.

Conclusions and Open Questions

$>$ Standardization of imaging procedures and performances/quantification is needed.

$>$ Intra-subject standardization, i.e., use of the same machine in the exact same way per patient, is essential and feasible in response assessment trials.

$>$ Inter-institute harmonization is needed occasionally, depending on the purpose of imaging within trial, but considered as difficult or even not feasible.

$>$ Standardization is challenged by ongoing technological advances.

$\triangleright$ Central image analysis and processing (for reaching harmonized results) may be the solution for now.

$>$ Imaging reports need to be standardized as well (i.e., use same annotations and language).

$>$ Despite the use of non-standardized imaging procedures, imaging has made a significant contribution for improving patient diagnosis with a direct impact on patient management.

\section{BS 6: Quantitative PET and MRI}

When combined in an integrated system, both PET and MRI should maintain their ability to quantify metabolic pathways and physiologic processes in vivo. Therefore, combined PET/MRI technology requires proof of limited cross-talk effects of the two imaging sub-systems. In sequential PET/MRI, the ability of PET to assess and quantify physiological and pharmacokinetic processes is not compromised by the MR component. In general, this also holds true for fully integrated PET/MRI systems [5]. However, due to the limited timing resolution of APDs, which are a constituent of MRI-compatible, integrated PET detectors today, time-of-flight emission imaging cannot be performed with the integrated PET/MR system available today.

Another factor related to the possibility of the PET sub-system of combined PET/MRI to deliver quantitative read-outs is the accuracy of the attenuation correction. Here the current approaches towards MR-AC employing tissue classification [3, 6]) may result in misclassifications and thus incorrect tracer uptake quantification. Further, neither of the three- or four-class MR-AC method does account for the presence of bone. This seems to result, for instance, for combined brain imaging, in a cranio-caudal gradient of the reconstructed PET tracer distribution, which is observed neither in PET nor in PET/CT images. Therefore, normal databases of stand-alone PET or PET/CT images should be used with caution when analyzing PET data from combined PET/MRI examinations. Further work is required for accurate PET quantification following MR-AC in combined PET/MRI, for instance, by incorporating bone information as obtained by ultra-short echo time MRI sequences or by employing artificial intelligence, like support-vector machine learning [15]. In addition, truncation artifacts arising from the limited axial field-of-view of the MR system can lead to incorrect quantification of whole-body PET tracer biodistribution with implications, for instance, in whole body PET tracer dosimetry [25]. Finally, the possibilities for image-derived input functions (IDIF) were discussed in the context of PET/MRI examinations. Access to an IDIF would help simplify full kinetic PET tracer modeling by avoiding invasive arterial blood sampling.

One example of an important research application of simultaneous PET/MRI is related to the possibility to crossevaluate quantitative read-outs obtained by both modalities in the clinical condition of acute ischemic stroke. Here it is now possible for the first time to examine the perfusion/ diffusion mismatch penumbra concept against $\left[{ }^{15} \mathrm{O}\right] \mathrm{H}_{2} \mathrm{O}$ $\mathrm{PET}$, the non-invasive in vivo gold standard for cerebral blood flow measurement (Fig. 4).

Another possibility to improve PET data quantification in combined PET/MRI relates to the option to detect organ movement during image acquisition. With simultaneous PET/MRI imaging, the correction of irregular movements of the urinary bladder or the gut during PET data acquisition using movement parameters derived from the simultaneously acquired MRI data becomes generally possible. Up to now, there is very limited evidence in the literature beyond respiratory movement correction using such methodology or simulations in animals or using phantoms.

Concerning MRI data, for sequential PET/MRI systems, the possibility to absolutely quantify the target parameter is not affected as long as radiofrequencies (RF) or susceptibility artifacts by the PET component do not disturb the MR signal detection. This also holds true for the integrated PET/MRI systems in which the PET detectors are placed between the RF 


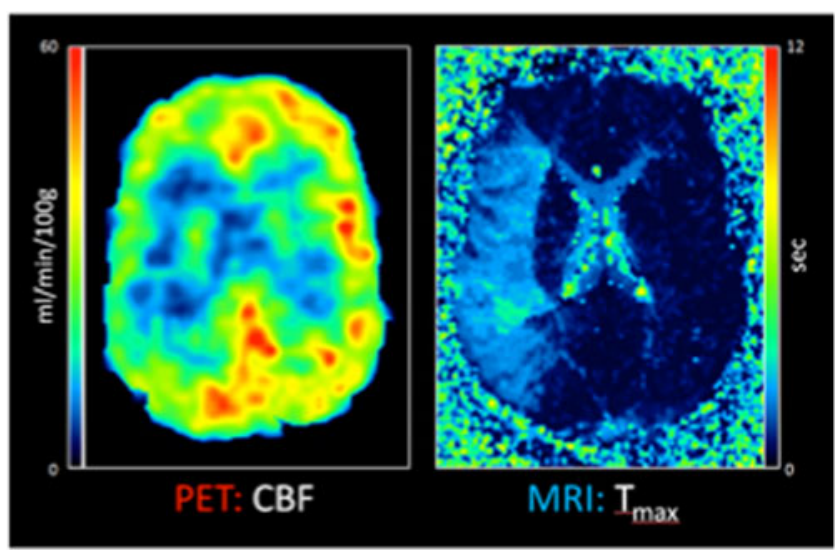

Fig. 4. Simultaneous quantification of cerebral blood flow $\left(\left[{ }^{15} \mathrm{O}\right] \mathrm{H}_{2} \mathrm{O}-\mathrm{PET}\right)$ and gadolinium bolus $T_{\max }$ (perfusion-weighted MRI) in a patient with acute ischemic stroke. Deficits in the right medial cerebral artery territory were found in this case in both modalities. A simultaneous imaging approach allows to cross-evaluate and calibrate new MR techniques of CBF determination in acute stroke against the gold standard $\left[{ }^{15} \mathrm{O}\right]$ $\mathrm{H}_{2} \mathrm{O}-\mathrm{PET}$. Data courtesy of the Department of Nuclear Medicine, University of Leipzig (Germany).

body coil and the gradient system. One drawback related to integrated PET/MRI systems is a smaller patient aperture $(60 \mathrm{~cm})$ as compared to PET/CT or stand-alone wide-bore $(70 \mathrm{~cm})$ MRI systems.

In summary, the quantitative performance of the PET component is not impaired by the MR component, and crosstalk effects are generally small, if at all noticeable in routine applications. As a current drawback, for the PET component of combined PET/MRI, the MRI-based AC methods currently employed lead to biased PET tracer uptake quantification.

BS 6. Quantitative PET and MRI

Key Questions

- Do the two sub-systems compromise each other in obtaining quantitative readouts?

- For the PET component of combined PET/MRI, is the tracer uptake quantification correct?

- How does the MR-based AC affect PET tracer uptake quantification?

- Are there ways to improve PET and MR readout quantification in combined PET/MRI?

Status Quo

- In combined PET/MRI, the quantitative performance of the PET component is not impaired by the MR component and vice versa.

- For the PET component of combined PET/MRI, the MR-based AC methods currently employed lead to suboptimal PET tracer uptake quantification.

- Combined PET/MRI may help derive suitable image-derived input function, with implications for simplifying PET tracer kinetic modeling.

- Combined PET/MRI offers to improve PET quantification by online motion correction using information obtained by MR techniques to track patient and organ motion.

Conclusions and Open Questions

$\triangleright$ Combined PET/MRI is suitable to cross-evaluate quantitative readout of both components.

$\triangleright$ More work is required to optimize MR-based PET data AC.

\section{Final Remarks}

The workshop attendees agreed that technological progress has led to the introduction of separate, co-planar and fully integrated PET/MRI design concepts for the sequential and quasi-simultaneous acquisition of multiparametric imaging data in clinical practice and research. Thus, combined PET/MRI will open without a doubt new perspectives in non-invasive imaging as part of clinical patient management and clinical research (Fig. 5). However, the current search for a single clinical "killer application" for PET/MRI continues and may eventually prove elusive. Even without such a specific "killer application", the convenience of easier patient handling, intrinsic image alignment, improved contrast and general versatility render PET/MRI desirable for clinical practice as well as research. Without a doubt PET/MRI already adds enormous value to pre-clinical studies of rodents.

However, numerous technological and methodological issues, as well as questions of standardization, have to be addressed before prospective multi-center studies help explore the clinical potential of PET/MRI.

Additional conclusions drawn from the discussions among the workshop attendees were, in short:

$\gg$ PET/MRI instrumentation and design concepts are workin-progress (WIP) with evidence of a steadily growing installed base of PET/MRI systems.

$>$ The full scope of quantitative and dynamic imaging functionalities must be maintained in combined PET/ MRI.

$>$ MRI-based attenuation correction is a WIP offering reasonable accuracy but limited reproducibility today.

$>$ MRI-based motion correction is a WIP without a readily applicable clinical implementation yet.

$>$ Requirements on the accuracy of PET/MRI will be renegotiated for specific applications.

$\gg$ Pre-clinical PET/MRI is far more advanced than clinical PET/MRI.

$>$ There is an immediate need for pro-active sharing of know-how among imaging producers and image users.

$>$ In view of a potential clinical adoption of PET/MRI, costeffectiveness studies need to be supported, considering the wide range or national reimbursement policies.

Naturally, the selection of the topics for the individual breakout sessions was limited to the preferences and needs felt by the organizers of the meeting and the faculty. 


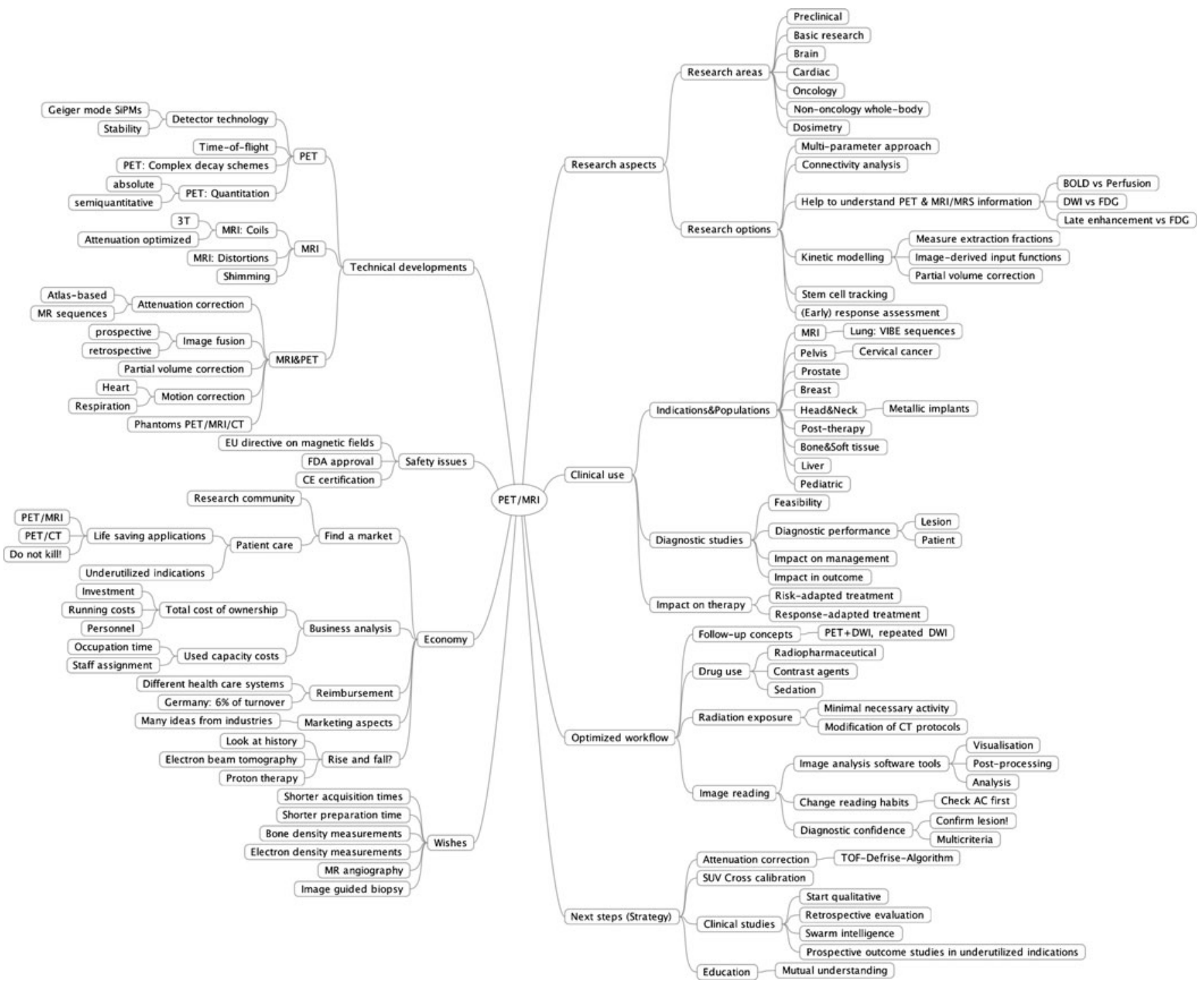

Fig. 5. "MindMap" as presented in the concluding remarks (Dirk Hellwig) illustrating the multiple options of PET, MR, and PET/MRI applications and implications for applications in clinical routine and research.

Likewise, the references provided in this summary report are limited to publications available at the time of the workshop; the bibliography was kept to an absolute minimum without giving credit to the rapidly expanding database of PET/MRrelated publications over the past year.

Following the successful First PET/MRI workshop 2012, we agreed to start an annual follow-up series of this application-driven educational event (for more information, see www.pet-mr-tuebingen.de). The authors are looking forward to hearing how the field of multimodality imaging continues to develop at the Second
PET/MRI Workshop, which will take place in Tübingen on April 8-12, 2013.

Acknowledgments. We would like to thank all participants for their active contribution at the 1st Tübingen Workshop on PET/MRI and for the lively discussions. The workshop was endorsed by the following societies: European Cooperation in Science and Technology (COST), European Society of Magnetic Resonance in Medicine and Biology (ESMRMB), European Society of Molecular Imaging (ESMI), World Molecular Imaging Society (WMIS), German Society of Nuclear Medicine (DGN), and German Society of Radiology (DGR). We would further like to acknowledge the generous support of the workshop sponsors: ABX-CRO, Hermes Medical Solutions, Mediso Medical Imaging Systems, Mirada Medical, Philips, П.pmod Biomedical Image Quantification, and Siemens Healthcare. 
Conflict of Interest. TB is a founder and owner of cmi-experts $\mathrm{GmbH}$, Switzerland. The Department of Preclinical Imaging and Radiopharmacy, University of Tübingen, is the European Preclinical Reference and Trainings Site of Siemens Healthcare. The department has ongoing research collaborations with Siemens and Bruker and licensed patents and inventions related to PET/MRI.

Open Access This article is distributed under the terms of the Creative Commons Attribution License which permits any use, distribution, and reproduction in any medium, provided the original author(s) and the source are credited.

\section{References}

1. Pichler BJ, Kolb A, Nägele T et al (2010) PET/MRI: paving the way for the next generation of clinical multimodality imaging applications. J Nucl Med 51(3):333-6

2. Beyer T, Freudenberg LS, Czernin J et al (2011) The future of hybrid imaging-part 3: PET/MR, small-animal imaging and beyond. Insights Imaging 2(3):235-246

3. Martinez-Möller A, Souvatzoglou M, Delso G et al (2009) Tissue classification as a potential approach for attenuation correction in wholebody PET/MRI: evaluation with PET/CT data. J Nucl Med 50(4):520-6

4. Zenge MO, Vogt FM, Brauck K et al (2006) High-resolution continuously acquired peripheral MR angiography featuring partial parallel imaging GRAPPA. Magn Reson Med 56(4):859-65

5. Delso G, Fürst S, Jakoby B et al (2011) Performance measurements of the Siemens mMR integrated whole-body PET/MR scanner. J Nucl Med 52(12):1914-22

6. Schulz V, Torres-Espallardo I, Renisch S et al (2011) Automatic, threesegment, MR-based attenuation correction for whole-body PET/MR data. Eur J Nucl Med Mol Imaging 38(1):138-52

7. Shao Y, Cherry SR, Farahani K et al (1997) Simultaneous PET and MR imaging. Phys Med Biol 42(10):1965-70

8. Slates RB, Farahani K, Shao Y et al (1999) A study of artefacts in simultaneous PET and MR imaging using a prototype MR compatible PET scanner. Phys Med Biol 44(8):2015-27

9. Marsden PK, Strul D, Keevil SF et al (2002) Simultaneous PET and NMR. Br J Radiol 75(Spec No):S53-9

10. Wehrl HF, Judenhofer MS, Wiehr S et al (2009) Pre-clinical PET/MR: technological advances and new perspectives in biomedical research. Eur J Nucl Med Mol Imaging 36(Suppl 1):56-68

11. Pichler B, Lorenz E, Mirzoyan R et al. (1997) Performance test of a LSO-APD PET module in a 9.4 tesla magnet. Nuclear Science
Symposium, 1997. IEEE Date of Conference: 9-15 Nov 1997, Volume: 2 Page(s): 1237-1239

12. Boone JM, Velazquez O, Cherry SR (2004) Small-animal X-ray dose from micro-CT. Mol Imaging 3(3):149-58

13. Badea CT, Drangova M, Holdsworth DW et al (2008) In vivo small animal imaging using micro-CT and digital subtraction angiography. Phys Med Biol 53(19):319-350

14. Funk T, Sun M, Hasegawa BH (2004) Radiation dose estimate in small animal SPECT and PET. Med Phys 31(9):2680-6

15. Hofmann M, Pichler B, Schölkopf B et al (2009) Towards quantitative PET/MRI: a review of MR-based attenuation correction techniques. Eur J Nucl Med Mol Imaging 36(Suppl 1):S93-104

16. Defrise M, Rezaei A, Nuyts J (2012) Time-of-flight PET data determine the attenuation sinogram up to a constant. Phys Med Biol 57(4):885-99

17. Therasse P, Eisenhauer EA, Verweij J (2006) RECIST revisited: a review of validation studies on tumour assessment. Eur J Cancer 42(8):1031-9

18. Eisenhauer EA, Therasse P, Bogaerts J et al (2009) New response evaluation criteria in solid tumours: revised RECIST guideline (version 1.1). Eur J Cancer 45(2):228-47

19. Young H, Baum R, Cremerius U et al (1999) Measurement of clinical and subclinical tumour response using [18 F]-fluorodeoxyglucose and positron emission tomography: review and 1999 EORTC recommendations. European Organization for Research and Treatment of Cancer (EORTC) PET Study Group. Eur J Cancer 35(13):1773-82

20. Wahl RL, Jacene H, Kasamon Y et al (2009) From RECIST to PERCIST: evolving considerations for PET response criteria in solid tumors. J of Nucl Med 50(5):122S-50S

21. Boellaard R (2009) Standards for PET image acquisition and quantitative data analysis. J Nucl Med 50(Suppl 1):11S-20S

22. Boellaard R (2011) Need for standardization of 18 F-FDG PET/CT for treatment response assessments. J Nucl Med 52(Suppl 2):93S$100 \mathrm{~S}$

23. Beyer T, Czernin J, Freudenberg LS (2011) Variations in clinical PET/ CT operations: results of an international survey of active PET/CT users. J Nucl Med 52(2):303-10

24. Herder GJ, Kramer H, Hoekstra OS et al (2006) Traditional versus upfront [18 F] fluorodeoxyglucose-positron emission tomography staging of non-small-cell lung cancer: a Dutch cooperative randomized study. J Clin Oncol 24(12):1800-6

25. Delso G, Martinez-Möller A, Bundschuh RA et al (2010) The effect of limited MR field of view in MR/PET attenuation correction. Med Phys 37(6):2804-12 